\title{
A WIRELESS SENSOR NETWORK SYSTEM DEPLOYMENT FOR DETECTING STICK SLIP MOTION IN GLACIERS
}

\author{
K. Martinez*, P.J. Basford*, D. De Jager*, J.K. Hart † \\ *Electronics and Computer Science, University of Southampton. UK, [km,pjb08r,ddj07r]@ecs.soton.ac.uk
}

†Geography and Environment, University of Southampton, UK, jhart@soton.ac.uk

Keywords: Glaciers, Environmental sensor networks

\begin{abstract}
The behaviour of glaciers is an area in which only limited research has been carried out due to the difficulties of monitoring sub-glacial movements. The authors believe that this can be addressed by the deployment of a wireless sensor network, consisting of heterogeneous sensors to instrument this activity. By deploying a sensor network measurements can be taken for a longer period than would otherwise be possible. The initial designs for this sensor network are presented along with details of some of the challenges posed by the project.
\end{abstract}

\section{Introduction}

The current understanding of how glaciers and ice sheets respond to climate change is extremely limited, and current numerical models have failed to predict that rapid ice losses that have been observed [4]. These models have failed because the accurate parameterisation of the basal boundary conditions of glaciers is still a very challenging area.

Recent studies of glacier movement have indicated that the movement is commonly episodic, and is known as 'stickslip' motion. Carrying out empirical studies into sub-glacial movement and behaviour poses lots of logistical challenges meaning that the number of projects investigating this is limited. In order to address these challenges, this project will apply lessons learnt from previous Glacsweb deployments [1] of sensor networks on glaciers. In this project, sub-glacial probes will measure the 'stick' phase while differential GPS (dGPS) and accelerometer readings will be used to measure the 'slip' phase.

The challenges involved in deploying such a sensor network include low power design, electronic hardware size and communication choices. However, these challenges are compounded by those brought in by the environment and the specific user requirements. These include the extreme environmental conditions (weather, pressures, stresses), remote location and logistical issues. In order to make a system which operates unattended over a long period of time and produces the data users need, a detailed understanding of system issues and risks are required. This understanding comes from both previous Glacsweb deployments [1, 2, 3], and understanding of how other environmental sensor networks have overcome the challenges.

\section{Related Work}

Geophones have previously been used to monitor glacial movement; one example is [5] in which the data from geophones with a frequency response peak of $12 \mathrm{~Hz}$ are continually sampled. Whilst in an ideal situation all data could be stored for later analysis, limitations of the deployment environment mean that this is not possible. This project uses techniques adapted from those used to monitor seismic activity around volcanoes [6]. This approach continually monitors the sensors and only when 'interesting' data is detected it is stored into memory, otherwise the unimportant data is discarded.

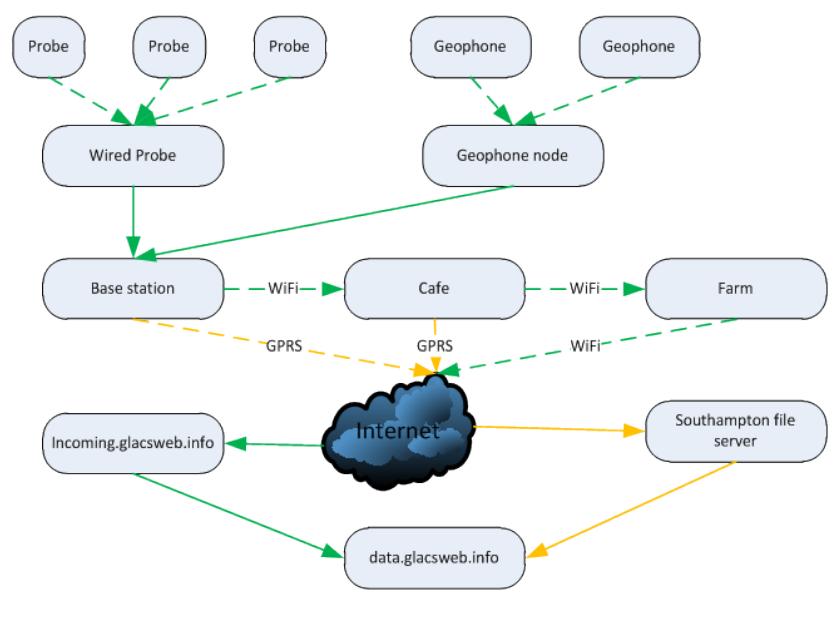

Primary Data Path Secondary Data Path

Figure 1: Overall system architecture

\section{Deployment environment}

This project's deployment site is the Skalafellsjökull glacier in Iceland. Skalafellsjökull is part of the largest ice-cap in Europe and provides a large area of accessible ice for the system to be deployed on. The area of the glacier used has good road links providing easy access to the glacier and deployment site. There is also mobile phone coverage which is used as one means of data transfer. As well as using the GPRS network previous Glacsweb trips have started to deploy the system infrastructure required in order to enable the collection of larger amounts of data than have previous been obtained. A further advantage is good access to local buildings and other infrastructure $1 \mathrm{~km}$ away providing 
mounting points for further wireless communications and dGPS reference points. However, the area of ice used is part of a branch of the glacier which means it moves slower than the main flow. In this case it moves approximately 6 meters per year, as opposed to the 25 meters that the main flow is estimated to move.

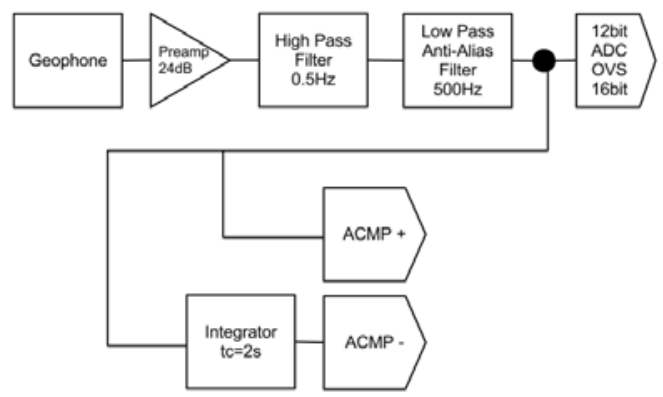

Figure 2: Overview of geophone hardware filtering

\section{System Architecture}

The system will make use of the $2.4 \mathrm{GHz}$ WiFi link that has been installed by a previous Glacsweb trip. This link provides a high-bandwidth connection to a nearby farm house with an internet connection. As there is no line of sight available from the ice surface the reference station (also the fixed location for the dGPS readings) acts as a relay for the WiFi connection. The system will also use GPRS as a backup data connection as previous experience has shown that the extreme environment requires multiple communication redundancies. Additionally the system is configured to send the data to one of several servers, in order to minimise the potential damage caused by server failure, this robust architecture is summarised in Figure 1. The WiFi connection enables remote access and configuration of the control systems over ssh. Due to the high power requirements of WiFi these subsystems are only powered up when needed. The data generated by all the sensors is collated by the base station which in turn handles all the external communication via either GPRS or WiFi. For this deployment the basestation is a BeagleBone running Ubuntu attached to a customdeveloped 'Ice-cape' printed circuit board which will provide the power switching and low power processing. This system is based on the Gumsense [2], however it has been updated to provide more functionality than the previous Gumstix connex based system. The main data collection will be facilitated by 3 types of sensing systems: probes, geophones and dGPS.

The probes will be deployed in the ice, the ice-till interface and the till layer (sediment under the ice). In a change from previous PIC and MSP430 based probes the 2012 version of the probes will be based around an ARM Cortex-M3 processor, this change has the advantage of providing access to a wide range of code libraries, a 32-bit instruction set and more low-power RAM. One outcome of this is that every probe will now have a microSD card which means that all data can be retained rather than being stored in a ring-buffer as in past deployments. This means that if periods of extended disconnection occur, no data is ever lost.

These probes are used to measure parameters such as waterpressure, temperature, and probe-movement using an accelerometer and compass. The compass and accelerometer will not be used to detect seismic events. In order to detect seismic events a second type of sensing system that will be used, this sensor will be based on the standard probe node with additional sensors added. The additional sensors that will be added are Geophones with a resonant frequency of $28 \mathrm{~Hz}$. These sensors will allow the sudden movements of the glacier to be detected through low-frequency oscillation. However despite the addition of microSD cards instead of using $512 \mathrm{kB}$ of EEPROM there is still not enough memory to record the output of the geophones continually. This means that the data will have to be processed locally on the node to detect events.

The signal will be filtered in hardware (Figure 2) and will trigger the low power analogue comparator; meaning that the MCU can remain in a low power mode until an event is detected. During this low power mode the ADC writes the incoming signal into RAM using DMA. Once an event has been triggered the contents of the RAM are written to the microSD card. The low power mode that the system normally operates in is predicted to consume $300 \mu \mathrm{A}$, and when it is writing the data out it is predicted to use $2.78 \mathrm{~mA}$. In order to capture the full event approximately $0.5 \mathrm{~s}$ of data before the event that triggers the capture is also recorded.

The final sensing system is dGPS, this is used in conjunction with the power management strategies presented in [3]. In the previous deployments, minimal dGPS data was collected. To detect 'stick-slip' behaviour longer and therefore more accurate periods of dGPS measurements are required in this deployment under severe power restrictions In order to make the data more manageable the GPS sampling rate is reduced from once per second to once every fifteen seconds. This will still produce over $4 \mathrm{MB}$ per day, which is why the highbandwidth WiFi link is required. The lowest level of dGPS recording will be for a continuous period of 2 hours. Whilst this will not provide the resolution of continuous data recording it will significantly reduce power usage, whilst still providing useful data.

This system thus uses the latest design and engineering solutions from previous deployments in order to successfully integrate new sensing requirements.

\section{Analysis of existing data}

Previous deployments of Glacsweb probes in Iceland have provided sub-glacial data for a period of two years, from 2008-2010. This has enabled a series of suspected 'stick-slip' events to be detected when the surface temperature rose above $2.5^{\circ} \mathrm{C}$. Figure 3 shows the accompanied falls in water pressure and movement in the probe. This data has been interpreted as the melt-water lubricating the bed of the glacier which allows the slip phase of the motion to occur. It is anticipated that new results from this deployment will allow 


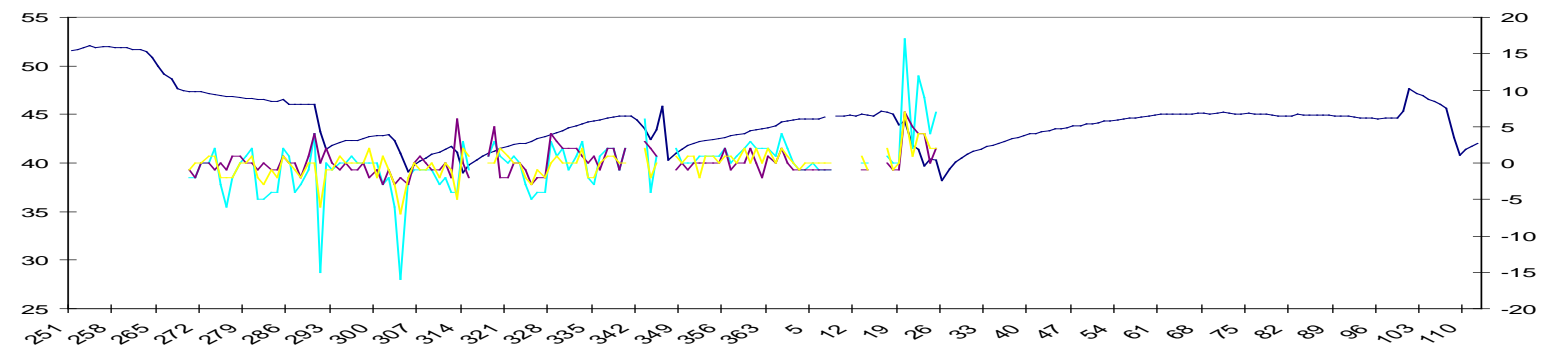

Figure 3: Probe results - Autumn/winter 2009/10 - water pressure (dark blue) (m water equivalent), change in tilt (3 axes, red, yellow and light blue)

the development of a model to predict when seismic events occur. This model could be used to change the system behaviour in the run-up to an event, such as increasing probe sampling intervals.

Surface geophones sampling at $1 \mathrm{kHz}$ were deployed in 2011 in order to evaluate the nature of the data that these sensors obtain. This showed that the sampling frequency could be reduced to $500 \mathrm{~Hz}$ without loss of information.

\section{Conclusions}

The use of environmental sensor networks can dramatically improve current knowledge of the way glaciers move and behave. The current area of focus in the Glacsweb project is to use these networks to monitor 'stick-slip' behaviour of glaciers. This will be achieved by using a heterogeneous sensor network consisting of: probes in the ice and till layers, geophones embedded within the ice and a differential GPS network in order to produce precise measurements of movement distance. As continual collection of data is too power intensive to be achieved in the long term, the GPS readings are scaled according to available power resources, and only geophone readings of interest are recorded. This data is then collated by the base station and sent daily to servers in Southampton for analysis. This transfer of data off the glacier is primarily through a WiFi network which has been deployed by a previous Glacsweb field trip, however, a backup GPRS link is also provided for when the WiFi link fails.

Data from previous sensor deployments in the glacier shows the potential for detecting these 'stick slip' events, and it is hoped that increased understanding of how the glacier moves will enable previous data to be re-examined for evidence of stick-slip motion.

\section{Acknowledgements}

This project is currently funded by the Leverhulme Trust.

\section{References}

[1] K. Martinez, P. Padhy, A. Elsaify, G. Zou, A. Riddoch, J. K. Hart, H. L. R. Ong. "Deploying a Sensor Network in an Extreme Environment”, IEEE
Computer Society: Sensor Networks, Ubiquitous and Trustworthy Computing, , pp.186-193 (2006).

[2] K. Martinez, P. Basford, J. Ellul, R. Spanton. "Gumsense - a high power low power sensor node", 6th European Conference on Wireless Sensor Networks (2009).

[3] K. Martinez, P. Basford. "Robust wireless sensor network performance analysis”, IEEE Sensors 2011 pp. 203-206 (2011).

[4] S. Solomon, D. Qin, M. Manning, Z. Chen, M. Marquis, K. B. Averyt, M. Tignor H. L. Miller (eds.). "The Physical Science Basis. Contribution of Working Group I to the Fourth Assessment Report of the Intergovernmental Panel on Climate Change”, IPCC 2007. Climate Change 2007, pp 996. (2007)

[5] F. Walter, N. Deichmann, M. Funk. "Basal icequakes during changing subglacial water pressures beneath Gornergletscher, Switzerland”, Journal Of Glaciology, 54, pp. 511-521, (2008)

[6] G. Werner-Allen, K. Lorincz, M. Welsh, O. Marcillo, J. Johnson, M. Ruiz, J. Lees, "Deploying a wireless sensor network on an active volcano”, IEEE Internet Computing, 10, pp. 18-25, (2006). 